\title{
REHABILITACIÓN Y REUTILIZACIÓN DEL PATRIMONIO URBANO AL SERVICIO DE LAS FUNCIONES DE LA CAPITALIDAD AUTONÓMICA. ANÁLISIS COMPARADO DE TOLEDO Y PAMPLONA ${ }^{1}$
}

\author{
Ana Eulalia Aparicio Guerrero \\ José María Martínez Navarro \\ Universidad de Castilla La Mancha \\ Ana.Aparicio@uclm.es ; JoseMaria.Martinez@uclm.es
}

\section{RESUMEN}

La nueva configuración político-administrativa del Estado, con la creación del sistema autonómico español, ha supuesto la mayor capacidad de las nuevas capitales para captar recursos y promocionarse. En las comunidades que abarcan varias provincias, las capitales se han resituado en el sistema urbano regional produciéndose la concentración de competencias en la capital y de funciones administrativas en sus centros históricos.

El objetivo de nuestro trabajo consiste en estudiar hasta qué punto el establecimiento de la capitalidad regional ha supuesto en estos espacios urbanos una potenciación de las políticas y actuaciones urbanas dirigidas a la rehabilitación y reutilización del patrimonio urbano al servicio de las funciones de capitalidad autonómica en Toledo y Pamplona.

Palabras clave: capitalidad autonómica, patrimonio urbano, rehabilitación, cambio de uso y regeneración.

Fecha de recepción: noviembre 2013.

Fecha de aceptación: noviembre 2014.

1 Este artículo se inscribe en el plan de difusión de resultados del Proyecto de Investigación «Las ciudades españolas en la etapa autonómica. Dinámicas, procesos y políticas (1978-2012) (URBSPAIN)», financiado por el Ministerio de Economía y Competitividad en el marco del Plan Nacional I+D+i (Código: CSO 2009-11261-Subprograma GEOG). El investigador principal del proyecto ha sido el Dr. D. Manuel Valenzuela Rubio, de la Universidad Autónoma de Madrid. 


\section{ABSTRACT}

Since the autonomous Spanish system was set up, the new political and administrative organization of the State, meant extending the new autonomous capitals capacity to raise funds and to promote them. In Autonomous Communities, each of which covers various neighboring provinces, the capitals have relocated into the urban regional system thus producing the concentration of power in the capital and of administrative functions in its historical centers.

The aim of our contribution consists of studying to what extent the establishment of the regional capitals have led to an empowerment of the policies and urban performances directed to rehabilitation and re-use of the urban heritage seeking to serve the functions of autonomous capital in Toledo and Pamplona.

Keywords: Autonomous capital, urban heritage, rehabilitation, change of use and regeneration.

\section{INTRODUCCIÓN}

En el marco del paradigma nacional-patrimonial (Bobbio, 1992), el patrimonio y las capitales mantienen una relación estructural y dialéctica, que en esta aportación podemos hacer extensiva a la construcción de la identidad territorial regional. El patrimonio, conjunto de bienes considerados dignos de pasar a la posteridad, constituido como el principal componente de la iconografía identitaria territorial, legitima las funciones de capital. Por su parte, la condición de capital, sede del poder político, induce un proceso de patrimonialización, mediante el cual un grupo social extrae un objeto de la vida social normal para atribuirle el estatus de emblema de su identidad en el tiempo (Micoud, 2005), que a su vez es parte de la producción de una comunidad nacional imaginada (Anderson, 1996) -regional en este caso-. Erigido en aparato ideológico del Estado (Althusser, 1976), el patrimonio participa de las funciones políticas -los lugares de poder son frecuentemente los lugares patrimonializados-, de las funciones de representación y de las funciones culturales de la capital, escaparate, emblema y supuesto crisol de la nación/región. De hecho, en algunos casos la cuestión del patrimonio y su utilización simbólica se ha complicado todavía más por la paradójica noción del patrimonio reconstruido (si aceptamos que la transmisión del patrimonio se justifica en el pasado) en ciudades como Dresde o Berlín (Voisin, 2007; Djament-Tran, 2013).

El objetivo de nuestra investigación consiste en analizar hasta qué punto el estatus de capitalidad ha supuesto la potenciación de las políticas e intervenciones dirigidas a la revitalización del patrimonio urbano al servicio de esas nuevas funciones de capitalidad. La amplia diversidad de capitales regionales resulta inabordable con las limitaciones de tiempo y medios materiales de que disponemos para verificar estos objetivos, por lo que nos hemos visto obligados a establecer una estricta selección de dos estudios de caso a partir de los siguientes criterios: que las dos ciudades seleccionadas respondan a categorías contrastadas dentro de la tipología de capitales regionales, que su base económica sea distinta y que ambas ciudades dispongan de órganos de gestión diferentes. Como consecuen- 
cia de la aplicación de estos criterios hemos seleccionado dos ciudades: Pamplona, capital de una comunidad Foral, y Toledo, capital de una región constituida por cinco provincias.

En el caso que nos ocupa, la comparación entre los dos estudios de caso seleccionados parece demostrar que Pamplona, que ya antes del estado de las autonomías ejercía su función de capital provincial y de capital simbólica de Navarra (heredera histórica del antiguo reino de Navarra, vigente hasta 1841 y del cual emana su particular régimen de autogobierno, denominado Régimen Foral), núcleo rector y organizador indisputado del territorio regional, opta por un modelo de rehabilitación y reutilización del patrimonio urbano selectivo, en el que aparte del Parlamento de Navarra, el Palacio de los Reyes de Navarra (hoy Archivo General de Navarra) y el Palacio de Congresos y Auditorio Baluarte, buena parte del resto de las dependencias administrativas de la Comunidad Foral y las dos universidades, privada y pública, optan por localizaciones relativamente periféricas y edificios de arquitectura contemporánea. Sin embargo, en el caso de Toledo, capital de la comunidad de Castilla-La Mancha, una región cuya identidad territorial aún está en proceso de construcción, donde la elección de la propia ciudad no se realizó sin disputa con otros núcleos urbanos de la red regional, que ocupa el cuarto puesto regional por tamaño y dinámica demográfica, y cuya proximidad y buena accesibilidad a Madrid explican que una metodología de delimitación funcional residencia/trabajo la deje englobada dentro del área metropolitana madrileña, la decisión inicial apostara por la recuperación de viejos contenedores infrautilizados para alojar en ellos dependencias administrativas de la nueva Junta de Comunidades o de la propia universidad regional, logrando con ello avanzar en pos de dos objetivos, recuperar y revitalizar un espléndido pero abandonado casco histórico y respaldar simbólicamente la elección de Toledo y la identidad regional apoyados en el binomio patrimonio-capital. En definitiva, con la aprobación del estatuto de autonomía de la región se inauguraría una nueva era en las relaciones entre una capital «re-encontrada» (capital del antiguo reino visigodo de Toledo y una de las sedes de la corte del imperio bajo Carlos I) y un patrimonio redefinido, elegida como capital histórica -o head link (Spate, 1942)en nombre de una argumentación patrimonial, Toledo entraría en una fase de intenso reciclaje urbano y en un nuevo ciclo patrimonial, paralelo a una nueva etapa de mundialización y re-escalado (Brenner, 2004) que debilita el papel de la escala nacional en favor de las escalas metropolitanas y regionales, y un nuevo régimen turístico globalizado del patrimonio que tiende a erigir a este último en ventaja comparativa en el contexto de la competencia urbana.

\section{SITUACIÓN DE PARTIDA}

La ciudad de Pamplona, fundada en el 74 a.C. por el general romano Pompeyo, está situada al pie del paso más accesible del Pirineo occidental, por donde discurría la ruta de peregrinación medieval del Camino de Santiago. Emplazada en la parte más alta de una terraza sobre el río Arga, que divide la ciudad en dos zonas disimétricas y de desigual desarrollo, por ella discurren otros dos ríos, el Elorz y el Sadar. Durante el siglo XIX la ciudad ve aumentada su población, pero el control militar imposibilita tirar las murallas y edificar en los alrededores. Por ello la ciudad se compacta. La construcción del primer y segundo ensanches se produce en el período de incorporación de Navarra a la modernización agraria en 1884 y 1915. Por último, el tercer ensanche es fruto de la modernización industrial y terciaria de las últimas décadas del siglo XX. 
Según se recoge en el Programa Iniciativa Comunitaria Urban Pamplona 2001-2006 (2001), el centro histórico sufrió desde 1970 una acusada tendencia al despoblamiento que en los últimos años parece estacionarse e invertir su evolución alcanzando una cifra cercana a los 12.000 habitantes. En este contexto, y como en la mayor parte de las ciudades, el casco histórico de Pamplona se convierte en foco de marginalidad asociado a vivienda deteriorada, envejecimiento demográfico, rentas bajas, tráfico y consumo de drogas.

En el marco de una política urbanística compleja, la recuperación urbana presta especial atención a dos problemas relevantes en las ciudades históricas contemporáneas: la preservación de los usos residenciales y la regeneración ambiental de los espacios libres. La conversión de las antiguas zonas militares situadas en el centro de la ciudad en parques públicos y zonas verdes y la adecuación de espacios de esparcimiento a las afueras han hecho de Pamplona la ciudad española con más zonas verdes por habitante y la sexta de la Unión Europea.

Actualmente, Pamplona, y fundamentalmente la meseta en donde se asienta el casco histórico y los ensanches de la ciudad, es el centro de la ciudad funcional y el centro de decisión y de poder de la región urbana de Navarra. Es el centro político, administrativo, económico, financiero y comercial de Navarra, además de centro cultural. En 1998 Pamplona obtuvo la «carta de capitalidad» por la que se reconoce que el carácter que la ciudad de Pamplona tiene como capital de la Comunidad Foral de Navarra, de acuerdo con lo dispuesto en el artículo 8 de la Ley Orgánica 13/1982, de 10 de agosto, de Reintegración y Amejoramiento del Régimen Foral de Navarra (LORAFNA), confiere a su Ayuntamiento una singularidad cualitativa, respecto de otras entidades locales, desde el momento en que numerosos servicios de la ciudad son demandados y utilizados, además de por los vecinos, por un alto porcentaje de población de hecho, porcentaje formado por población flotante de su comarca y del resto de Navarra, y por residentes no inscritos en el padrón, que acuden a la capital como usuarios con carácter ocasional o permanente de los grandes servicios públicos que tanto la Administración Foral como la Estatal tienen implantados en Pamplona, precisamente por ser capital de la Comunidad Foral (Alli, 2001). Como Pamplona no puede financiar por los cauces normales esa sobredimensión de servicios que necesita, la carta de capitalidad tiene como objetivo dotar al Ayuntamiento de un complemento singular de financiación con cargo al presupuesto general de Navarra.

En cuanto a la segunda ciudad objeto de nuestro estudio, Toledo, ha sido desde la conquista romana y durante toda la Edad Media una de las ciudades más importantes de la Meseta por su situación estratégica en el centro del valle medio del Tajo y por sus características defensivas. Muchos de sus monumentos son obras maestras de distintas civilizaciones, creadas en un contexto en el que la presencia de tres grandes religiones, judaísmo, cristianismo e islamismo, ha constituido un factor esencial en sus trazados y construcciones.

Toledo, cuyo casco antiguo está situado en la margen derecha del río Tajo, tuvo que esperar a mediados del siglo XIX para comenzar la expansión fuera de las murallas; primero en los alrededores de la estación de ferrocarril para despegar definitivamente desde finales de los años 40 del siglo XX por la vega del Tajo.

En la actualidad Toledo, capital de la comunidad autónoma de Castilla-La Mancha desde 1983 y ciudad Patrimonio de la Humanidad desde 1986, tiene una configuración muy dispersa, existiendo barrios separados del núcleo principal de la ciudad por amplios espacios abiertos. El decrecimiento del peso específico poblacional del centro respecto a la ciudad es una tendencia que ha sido favorecida por la creación de espacios libres por demolición de 
Figura 1

PANORÁMICAS DE PAMPLONA

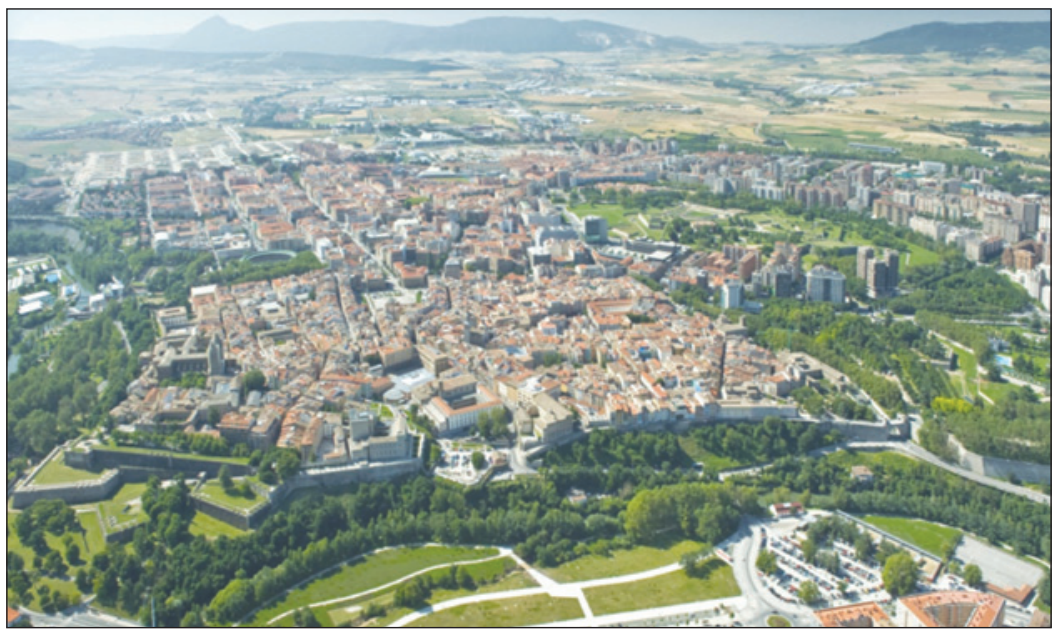

Fuente: http://www.fortiuspamplonabayonne.eu/index.php/pamplona/fortificaciones/pamplona-ciudad-fortaleza/

Figura 2

PANORÁMICA TOLEDO

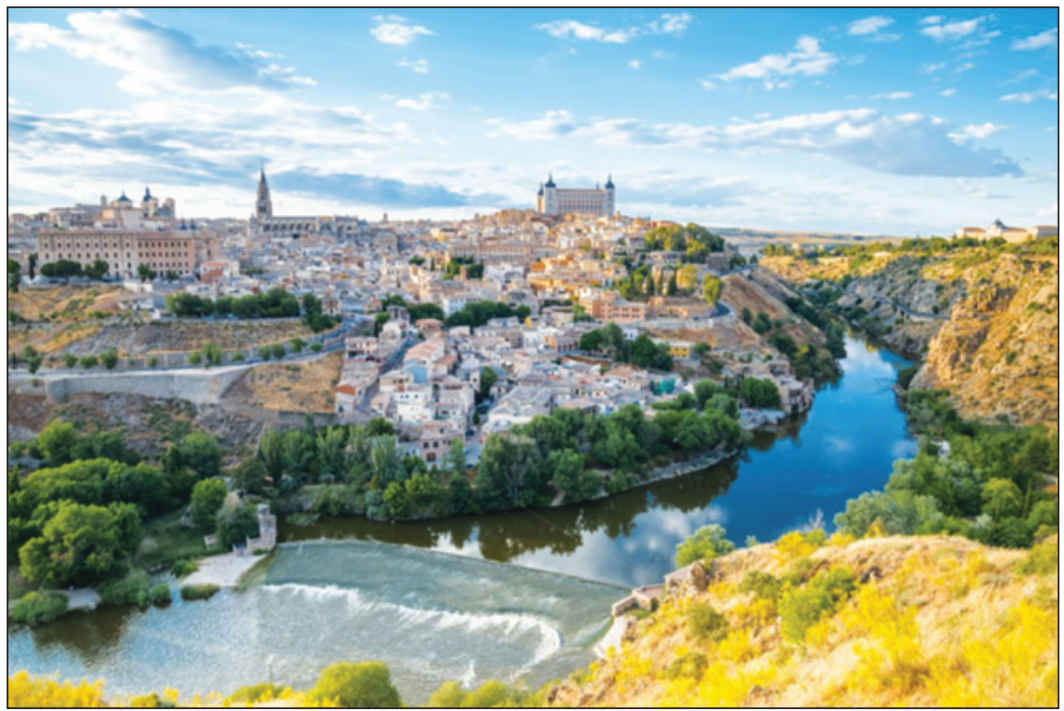

Fuente: http://blog.goeuro.es/excursiones-cerca-de-madrid/

espacios construidos, oferta de residencia en otros lugares de la ciudad en mejores condiciones que las del centro histórico e impulso del emplazamiento de instituciones administrativas dentro del casco, es decir, impulso a la localización de actividades administrativas en edificios monumentales a partir de 1982, cuando Toledo se convirtió en capital de la comunidad 
Figura 3

CASCO HISTÓRICO DE PAMPLONA

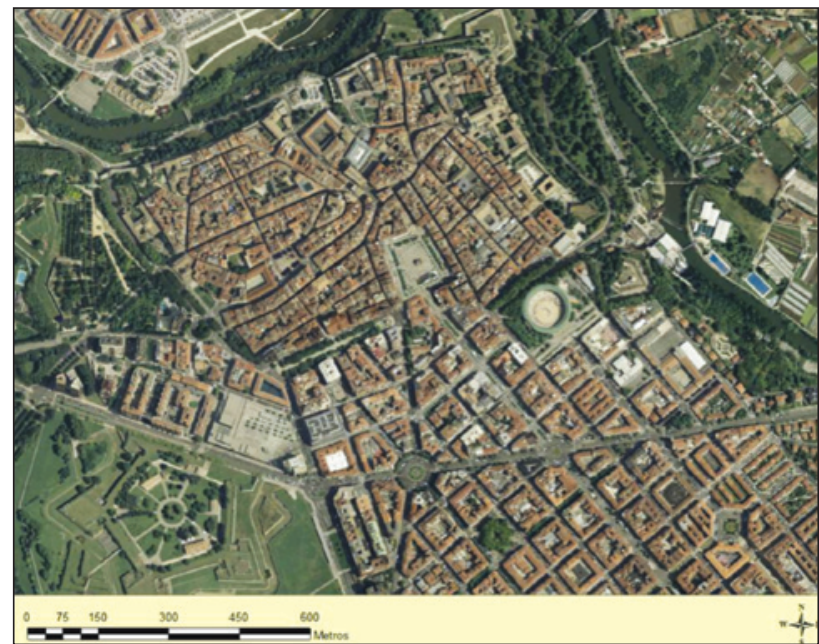

Fuente: elaboración propia.

Figura 4

CASCO HISTÓRICO DE TOLEDO

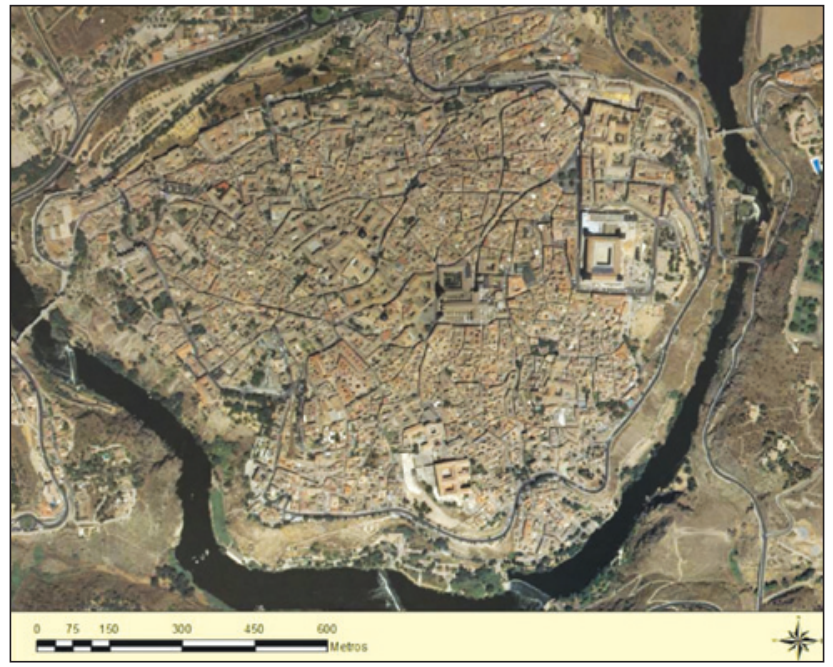

Fuente: elaboración propia.

autónoma, un proceso que ha supuesto la rehabilitación de numerosos edificios monumentales para nuevos usos administrativos y la rehabilitación del patrimonio residencial. La población disminuye hasta los 12.000 habitantes en los años noventa, en que comienza un momento de estabilización y una «cierta recuperación» en los años finales del siglo XX y comienzos del XXI (Almarcha et al., 2011). 
En definitiva, esta zona ha perdido prácticamente el uso residencial tradicional a raíz del deterioro de muchas viviendas, presentando una clarísima terciarización en relación con su carácter de capital regional y con el importante turismo que atrae esta zona de la ciudad, tanto por su propia configuración urbana como por los destacados monumentos históricos y artísticos que posee.

Los principales problemas que afectan a este espacio urbano son el progresivo vaciamiento de los inmuebles por la deficiente habitabilidad de las viviendas, el deterioro de algunos edificios históricos y la saturación y congestión de oficinas, equipamientos y comercios. Todo ello ha provocado un progresivo envejecimiento de los grupos sociales que viven en esta zona y la necesidad de buscar espacios más amplios en la periferia de la ciudad, fundamentalmente en la carretera N-400, para ubicar dependencias de la administración regional que en el casco eran ya totalmente inviables por la limitación de espacio. Todos estos problemas exigen, como en el caso de Pamplona, la existencia de políticas de rehabilitación, morfológica y funcional, que revitalicen este espacio urbano.

\section{PLANEAMIENTO URBANÍSTICO, POLÍTICAS URBANAS Y CENTROS HISTÓRICOS}

La planificación integral, consistente en una amplia gama de intervenciones en lo morfológico, estructural, residencial, monumental y funcional, constituye un elemento básico para el mantenimiento de los conjuntos históricos como ciudades vivas (Almarcha et al., 2011). Los catálogos y el planeamiento especial de protección, reforma o mejora, al permitir una visión global e integrada de los problemas del centro histórico, se configuran como los instrumentos fundamentales de la protección y recuperación urbana. En la práctica todavía son bastante pocos, no llegan a la centena, los centros históricos que cuentan con planeamiento especial aprobado, ello a pesar de las exigencias de la Ley de Patrimonio Histórico Español de 1985.

\section{III.1. Comunidad Foral de Navarra}

Navarra, al igual que el resto de las comunidades autónomas, tiene competencia exclusiva en materia de Ordenación del Territorio y Urbanismo así como de patrimonio monumental, histórico, artístico y arqueológico, siendo la Ley Foral 35/2002, de 20 de diciembre, de Ordenación del Territorio y Urbanismo y la Ley Foral 14/2005, de 22 de noviembre, del Patrimonio Cultural de Navarra, la normativa actualmente en vigor.

El Plan General de Ordenación Urbana (PGOU) de 1984 marca un punto de inflexión entre las lógicas del urbanismo desarrollista y las nuevas políticas orientadas a reformar, consolidar y recuperar la ciudad construida ${ }^{2}$. Este marco de cambio y austeridad propicia muchas actuaciones de rehabilitación de los viejos edificios en desuso para adaptarlos a las nuevas necesidades. Pero el PGOU se mostró incapaz de resolver los problemas de las áreas antiguas.

Ya en 1986 se aprobó el Plan Especial de Reforma Interior del Burgo de San Cernin, y posteriormente el Plan Especial de Reforma Interior del Casco Antiguo de Pamplona. En este último el ámbito considerado incluía la primitiva ciudad episcopal o Navarrería y la población de San Nicolás, que constituyen, junto al Burgo de San Cernin, el centro histórico de la ciudad de Pamplona (Ordeig-Corsini, 2011).

2 Plan Municipal de Pamplona. TEXTO REFUNDIDO. Aprobación Definitiva: Acuerdo C.O.T. 18/12/02 Memoria I-Análisis. 
El Plan Especial de Protección y Reforma Interior del Casco Antiguo (PEPRI) se presenta en 2001, siendo éste el que guíe a partir de entonces las intervenciones de la administración local a fin de superar el proceso de degradación social, económica y arquitectónica del barrio. El Plan Especial busca incentivar, a la vez que diversificar, las actividades centrales y de servicio en relación con otras ya instaladas en el casco, como la universitaria o la administrativa. Para ello se establecen áreas que admiten usos no residenciales, y no necesariamente comerciales (Campos, 1998). El PEPRI contempla una reducción de 1.500 viviendas aproximadamente (de las 6.533 viviendas se pasaría a unas 5.000) con el fin de producir un esponjamiento, liberando patios interiores de manzanas, y estableciendo una previsión de población de 14.000 habitantes. En la actualidad existen unas 1.300 viviendas vacías, un $21 \%$ de las existentes en este espacio.

El objetivo común que los redactores del Plan Municipal y del Plan Especial recogieron en ambos documentos consistía en reforzar la influencia y el lugar que ocupa Pamplona tanto en el ámbito regional como europeo y dotar al área de un planeamiento dirigido a su recuperación y conservación. Con la aplicación de sus ordenanzas se buscaba la permanencia de los valores tipológicos y morfológicos de la ciudad antigua, y también de aquellos usos y actividades que aseguraran un equilibrio social y funcional del área (Ordeig-Corsini, 2011).

Entre tanto, surgen dos organismos municipales que serán los que gestionen las actuaciones en este espacio: la Oficina Municipal de Rehabilitación en los años 80, y la empresa pública Pamplona Centro Histórico (PCH) en 2003 (Martínez Lorea, 2010). Las competencias de la Oficina Municipal de Rehabilitación se centran en el diseño y redacción de informes y estudios sobre planeamiento específico del Casco Antiguo, Primer y Segundo Ensanche, en la rehabilitación de edificios del casco antiguo y en general de toda la ciudad, y en el otorgamiento de las correspondientes licencias de obra. La misión de Pamplona Centro Histórico, sociedad pública participada al $100 \%$ por el Ayuntamiento de Pamplona, consiste en la realización de todas las actuaciones urbanísticas de edificación y de regeneración económica y social necesarias para la revitalización o rehabilitación integral de los espacios comprendidos en el ámbito del Casco Antiguo, Primer y Segundo Ensanche.

\section{III.2. Castilla-La Mancha}

Al igual que en Navarra, y sin perjuicio de lo dispuesto en la Constitución respecto a las competencias estatales, el Estatuto de Autonomía de Castilla-La Mancha establece para la Junta de Comunidades la competencia exclusiva en lo concerniente a la Ordenación del Territorio y Urbanismo, así como al patrimonio monumental, histórico, artístico y arqueológico. La Ley de Ordenación del Territorio y Urbanismo y la ley 4/1990 de 30 de mayo de Patrimonio Histórico de Castilla-La Mancha son las normas que regulan estos aspectos. Esta última representa un avance en la relación entre protección del patrimonio histórico y planeamiento urbanístico ya que en toda revisión del planeamiento urbanístico obliga a incluir catálogos de edificios monumentales y a definir con exactitud la zona considerada como entorno monumental y sus condiciones urbanísticas.

El Plan General Municipal de Ordenación urbana de Toledo de 1986 considera que el desarrollo del Conjunto Monumental debe estar regulado por un planeamiento especial, aunque tanto este como el anterior eluden el problema. En 1997, once años después de ser califi- 
cada por la UNESCO Patrimonio de la Humanidad, entra en vigor el Plan Especial del Casco Histórico. El Plan tiene como objetivos la proyección de Toledo como capital monumental con funciones culturales, representativas, residenciales, turísticas y administrativas, la puesta en valor del patrimonio existente como elemento activo de la ciudad y el fortalecimiento de la residencia como actividad básica del casco (Almarcha et al., 2011).

Años más tarde, en 2004, el Ayuntamiento de Toledo encomendó la redacción de un Plan de Ordenación Municipal (POM) que debía sustituir al Plan General de Ordenación Urbana de 1986. Así comienza a elaborarse un nuevo plan con una propuesta de ciudad unitaria en la que se quiere proyectar los nuevos crecimientos para llenar los vacíos del discontinuo urbano. El Plan, vigente desde 2007, proyectaba la expansión de la ciudad por las tierras llanas de la vega del Tajo, lo que produciría la desaparición de los paisajes que han contribuido a definir la personalidad de la ciudad (Zárate, 2007).

Por lo que respecta a la gestión y participación de las administraciones locales en la revitalización del casco histórico, Toledo es una de las tres ciudades patrimonio de la Humanidad que cuenta con una Real Fundación, un Real Patronato y un Consorcio, junto a Santiago de Compostela y Cuenca. La Real Fundación de Toledo, institución cultural privada constituida en 1988, tiene como fin la conservación y revitalización de la ciudad histórica de Toledo mediante actividades dirigidas a fomentar la habitabilidad de la ciudad y la protección, estudio y difusión de sus bienes patrimoniales. Diez años más tarde, la Real Fundación participó de la composición del Real Patronato de la ciudad de Toledo, con el fin de promover la ejecución de obras, servicios e instalaciones en general, de impulsar la coordinación de las inversiones públicas, favorecer la puesta en marcha de iniciativas culturales orientadas a la conservación del Patrimonio Histórico y potenciar la ciudad de Toledo como lugar de encuentro de culturas diversas (Troitiño, 2000). Además del apoyo administrativo y de gestión, el Real Patronato tiene como fines específicos el promover y facilitar la coordinación de las competencias de los distintos niveles administrativos, y el ejercer todas aquellas funciones que las administraciones consorciadas le encomienden. El ente gestor y administrativo del Real Patronato, el Consorcio de la ciudad de Toledo, se constituyó el 10 de enero de 2001 con carácter público, con personalidad jurídica propia y plena capacidad de obrar. En él están representados todos los niveles de la administración.

Las actuaciones realizadas durante estos catorce años de funcionamiento del Consorcio lo han convertido en el principal elemento dinamizador de la actividad rehabilitadora y revitalizadora del casco histórico de la capital de Castilla-La Mancha, siendo la referencia en todo lo relacionado con la puesta en valor del patrimonio toledano, tanto monumental como residencial.

Además, tras la experiencia adquirida en las intervenciones de rehabilitación en las diecinueve Áreas de Rehabilitación declaradas, como en el resto del casco histórico, el Ayuntamiento de Toledo declara en el año 2010 todo el casco histórico como una única Área de Rehabilitación Integral (ARI), simplificándose notablemente los trámites a realizar a la hora de solicitar subvenciones.

\section{REHABILITACIÓN Y REUTILIZACIÓN DEL PATRIMONIO URBANO COMO EXPRESIÓN DE LA NUEVA CAPITALIDAD POLÍTICA}

La Ley de Suelo aprobada mediante el Real Decreto Legislativo 2/2008, de 20 de junio conecta con los objetivos del Programa de Buenas Prácticas Urbanas y Liderazgo Local promovido por 
Naciones Unidas, cuando plantea la necesidad de recuperar la ciudad como creación cultural colectiva y favorecer su rehabilitación y el fomento de su uso (Corredor, 2009). La recuperación y reutilización productiva del patrimonio cultural, en el marco del reforzamiento de las centralidades administrativas, simbólicas, culturales y turísticas, requiere superar enfoques meramente arquitectónicos. La lectura del patrimonio cultural como fuente de riqueza y no sólo como de gasto, abre nuevas vías de acción a la hora de abordar las relaciones entre administración, cultura, turismo, comercio, etcétera. En este contexto es donde interesa situar el análisis y la valoración de las políticas de protección y recuperación de los centros históricos

\section{IV.1. Pamplona}

Con la recuperación de la democracia y la nueva estructura territorial autonómica, Pamplona se convierte en capital de la Comunidad Foral de Navarra y su Casco Histórico en un espacio susceptible de experimentar operaciones de rehabilitación y cambio de uso en contenedores infrautilizados para albergar alguna dependencia del aparato administrativo de la Comunidad Foral.

Se puede afirmar que en Pamplona la evolución de las actuaciones edificatorias y urbanísticas en el centro histórico ha seguido el proceso común a gran parte de las ciudades del entorno. Este proceso, que ha tenido lugar al amparo de políticas generales de rehabilitación a nivel estatal y regional, se ha traducido en el apoyo a las actuaciones privadas a través de diversas ayudas económicas directas y, si bien se ha revelado como imprescindible, durante los primeros años 90 ha iniciado una progresiva ralentización. Pero las ayudas estatales y autonómicas resultan insuficientes en situaciones de deterioro y contextos socioeconómicos de gran fragilidad; en estos casos son necesarias Ordenanzas Municipales de Ayudas a la Rehabilitación. Esta vía de acción es la puesta en marcha en Pamplona y en ciudades como Vitoria, Barcelona, Alcoy, Santiago de Compostela, etc., pero aún continua siendo, por la debilidad financiera de los ayuntamientos, una opción bastante minoritaria. Según los redactores del Texto Refundido del Plan Municipal, como consecuencia de la puesta en marcha de la Oficina de Rehabilitación y la aplicación de la Ordenanza Municipal de ayudas, se produjo en la segunda mitad de la década de los 80 una importante actividad rehabilitadora, tanto en el parque residencial como en el de los equipamientos: Oficina de Rehabilitación, Conservatorio Municipal, Oficinas Municipales, Edificio de Promoción Ciudadana, Plaza de la O, Mercado de Santo Domingo, Escuela de Idiomas, etc.

Pero los edificios remodelados de manera previa a la redacción del Plan Especial demostraron que esto no era suficiente, y el propio plan recogía como objetivo prioritario estructurar dicho conjunto con un sistema de espacios libres, accesos peatonales y rodados, que contribuyera por un lado a dar a la zona el carácter central, simbólico y representativo del casco antiguo y sirviera, por otro, como catalizador para la mejora y recuperación integral de esta parte fundamental de Pamplona. Respecto a los espacios libres, se adoptaron unos criterios homogéneos en cuanto a materiales, diseño de elementos de urbanización y mobiliario urbano, siendo el proyecto de urbanización emplazado frente al Palacio Real el que marcó las pautas que habían de seguirse en todo el conjunto (Ordeig-Corsini et al., 2011).

La segunda mitad de los 90 y el primer decenio de 2000 se caracterizan por la prosperidad económica. La administración comienza a acometer obras públicas, produciéndose en 
estos años la transformación más visible de este espacio. Es también la década de la Universidad Pública, que si bien podría haberse ubicado en contenedores del casco histórico, como se planteó en la capital castellano-manchega, se optó por un emplazamiento periférico a partir de la construcción de nuevas edificaciones33. Durante el período 1994-1999 los barrios Rochapea y Centro Histórico han sido objeto de algunas intervenciones a nivel local y regional que es preciso destacar. En el año 1996 se inicia el proceso de reurbanización y peatonalización con la instalación de una galería de servicios subterránea (gas, luz, agua, teléfono, basuras, etc.) en la zona sur, donde existe mayor presencia comercial, y se abordan importantes operaciones de rehabilitación de edificios y viviendas (Martínez Lorea, 2010). A su vez van surgiendo un conjunto de parkings subterráneos en los límites y dentro del propio barrio, entre los que destaca, por la fuerte polémica que generó, el de la Plaza del Castillo (años 2002-2003), situada en la conexión del Casco Antiguo con el Segundo Ensanche. Las ayudas del Gobierno de Navarra para rehabilitación de edificios y viviendas, vigentes hasta finales de 2012, provocaron un notable aumento de las rehabilitaciones en Pamplona hasta tal punto que el volumen de trabajo obligó a incrementar la plantilla de empleados de la Oficina de Rehabilitación del Ayuntamiento de Pamplona, que pasó de 11 a 17 personas (5 aparejadores, 2 arquitectos, 2 letradas, 6 administrativos, una secretaria técnica y un director).

Hay que esperar a los primeros años del milenio para que la administración empiece a ocuparse de los grandes edificios públicos. Según los autores del Catálogo Desarrollo Urbano 1950-2000 (2011), el ámbito de actuación del Plan Especial de Protección y Reforma Interior del Palacio Real (aprobado definitivamente en 1994), uno de los más singulares al contrastar con la compacta trama del casco, era considerado como una zona histórica de oportunidad (Ordeig-Corsini et al., 2011), ya que en ella coexistían amplios espacios libres con un conjunto de edificios singulares de grandes dimensiones que entonces estaban relativamente desaprovechados, pero que podían servir como contenedores para usos públicos o institucionales muy diversos. La propuesta tenía como objetivo principal crear las condiciones urbanísticas adecuadas y precisas para la rehabilitación y reutilización del Palacio Real y de todo su entorno. Por eso, ponía de manifiesto las grandes posibilidades de recuperación de la zona, de sus edificios singulares y su infrautilización. El plan adjudicaba nuevos usos a los diferentes edificios, algunos de ellos remodelados años antes dentro de las actuaciones planteadas por el Plan Especial del Casco Antiguo. Además, el Plan adelantaba que el antiguo Hospital Militar iba a ser reutilizado como sede del Departamento de Educación del Gobierno de Navarra, pero finalmente fue el Convento de Santo Domingo, en la calle del mismo nombre en el casco antiguo de Pamplona, el contenedor que albergaría este nuevo uso.

Un ejemplo de todo ello es la rehabilitación del edificio que años atrás, hasta 1996, fue sede del Palacio de Justicia, para instalar en él el Parlamento de Navarra, creado por el Amejoramiento de 1982 -que tras la democratización de las instituciones suponía la actualización del régimen foral conforme a la Constitución de 1978-. Está situado en el boulevard o Paseo de Sarasate del primer ensanche de Pamplona, que une el casco antiguo de la ciudad con la nueva Pamplona creada por el segundo ensanche (figuras 5 y 6), siendo la única construcción civil de carácter oficial que se levantó en el primer ensanche.

3 Diariodenavarra. es. Especiales. Arquitectura en Pamplona. Disponible en http://www.diariodenavarra.es/ especiales/arquitectura/index $\cdot$ asp? $\sec =\operatorname{anos} 90$ 
Figura 5

FACHADA PARLAMENTO DE NAVARRA

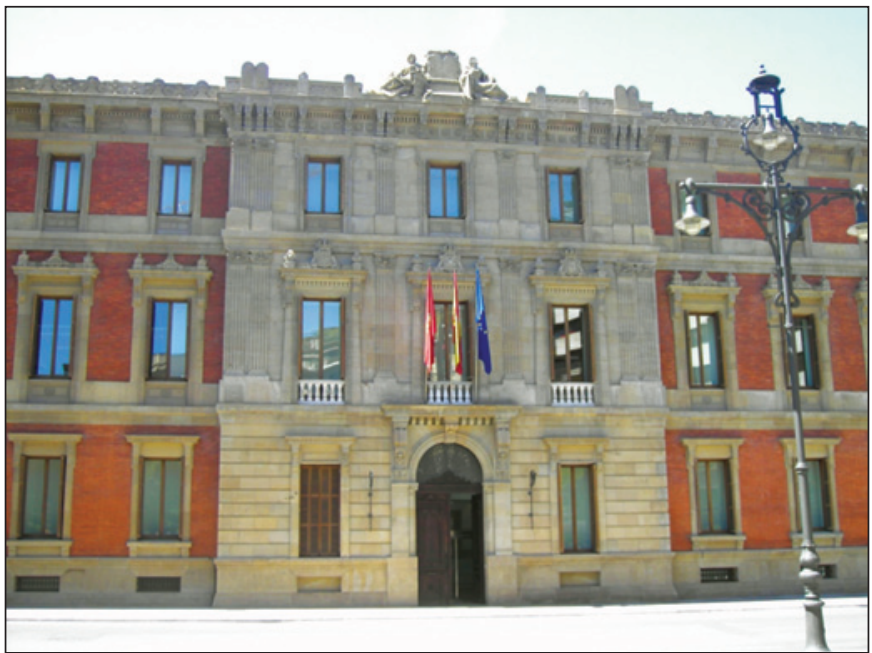

Fuente: http://www.esacademic.com/dic.nsf/eswiki/901361. Disponible en: http://www.esacademic.com/pictures/eswiki/78/NafarParlamentua.JPG

Figura 6

INTERIOR PARLAMENTO DE NAVARRA

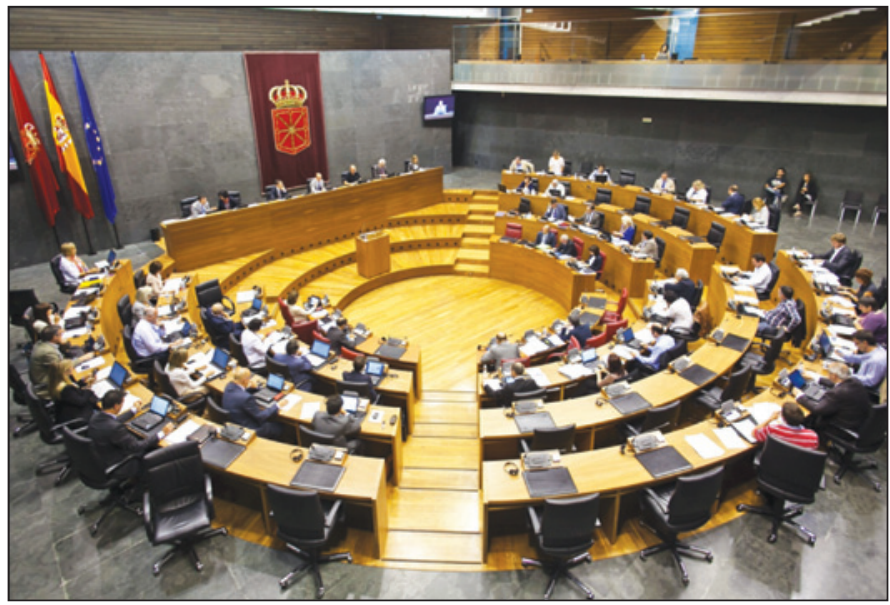

Fuente: Parlamento de Navarra. www.parlamentodenavarra.es. Disponible en: http://www.parlamentodenavarra.es/UserFiles/images/Noticias/Orig-027-9-12-1.jpg

El edificio, de finales del XIX, ocupa una parcela irregular por lo que las fachadas tienen distintas dimensiones y está formado por tres plantas con grandes ventanales y combinación del ladrillo rojo y la piedra ocre de Tafalla. El cuerpo principal se acerca al clasicismo con 
empleo exclusivo de la piedra. La intervención, comenzada en el 2002, se centró en el interior manteniendo las fachadas y la crujía perimetral. Las fachadas interiores del patio y el suelo de vidrio le dan un aspecto de modernidad que contrasta con las fachadas exteriores.

El cambio de siglo constituye, según Martínez Lorea (2010), un periodo de bonanza económica para las arcas municipales lo cual permite al Ayuntamiento optar por intervenciones de gran envergadura por su impacto arquitectónico en la ciudad y por ser diseñadas por dos arquitectos locales de prestigio internacional como son Rafael Moneo y Patxi Mangado: el Archivo Real y General de Navarra y el Palacio de Congresos Baluarte, icono de la cultura musical y teatral de la ciudad. El archivo se trasladó al Palacio de los Reyes, del siglo XII y ubicado en el casco antiguo de Pamplona, tras abandonar su sede en el Palacio de Diputación, situado en el primer ensanche (figuras 7 y 8). Tras la Conquista de Navarra en el siglo XVI el palacio pasó a ser la residencia de los Virreyes, posteriormente Capitanía General, Gobierno Militar hasta 1971 y tras un largo abandono, en 1999 se pone la primera piedra de su reconstrucción. La reforma terminó en el año 2003 (Alli, 2001). La intervención combina la reconstrucción de la L original del antiguo Palacio de los Virreyes de Navarra con la introducción de una poderosa volumetría, que desde el exterior da la imagen de una torre homenaje. La parte restaurada y la nueva se relacionan en el patio produciendo un lugar casi sin fecha, contemporáneo e «histórico» ${ }^{4}$.

Estos cambios de uso, junto con la construcción en el solar - aparcamiento de Padre Moret del Palacio de Congresos en el 2003 (figuras 9 y 10), han contribuido de forma importante a la revitalización de la zona. Se trata de un edificio en L que se cierra como una réplica del muro de la Ciudadela a la avenida del Ejército y abre su plaza a la ciudad.

Figura 7

ARCHIVO GENERAL DE NAVARRA

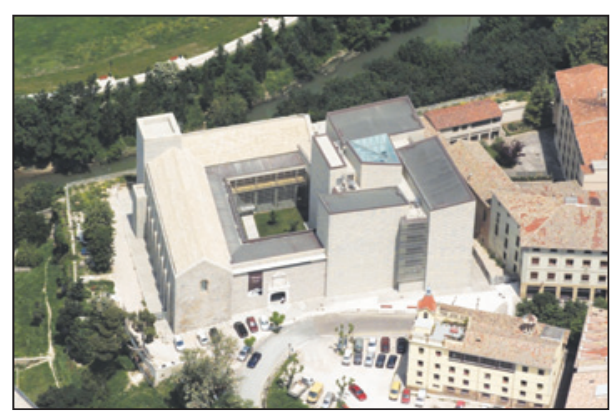

Fuente: Dirección General de cultura, www.navarra.es Imagen disponible en http://www.navarra.es/ NR/rdonlyres/644A12B4-25D9-43E8-AE1E1548673B8922/163341/ArchivoRealT002088.jpg
Figura 8 PATIO ARCHIVO GENERAL DE NAVARRA

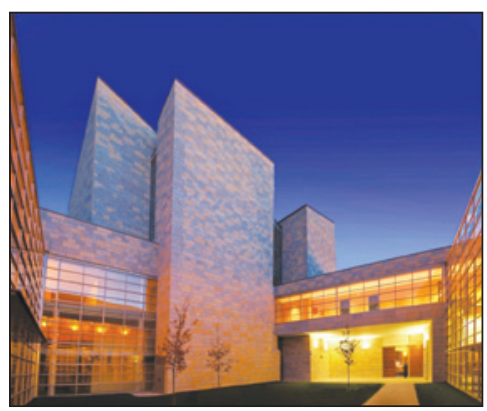

Fuente Miguel Urzainqui, (https://plus.google. com/+MiguelUrzainqui/posts) disponible en http://3.bp.blogspot.com/-2d4-1JdHq1k/VNeznq0kTdI/ AAAAAAAApwE/ssbEA7KqFLg/s1600/5.jpg

4 El Gobierno de Navarra edita el libro «El Palacio Real de Pamplona» sobre la historia de la sede del Archivo General. Martes, 14 de diciembre de 2004. Disponible en http://www.navarra.es/home_es/Actualidad/ Sala+de+prensa/Noticias/2004/12/14/1412cu60.htm. 
Figura 9

PALACIO DE CONGRESOS YAUDITORIO BALUARTE

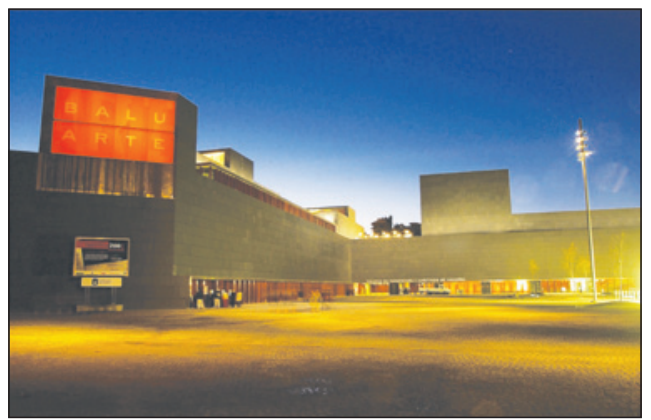

Fuente Ayuntamiento de Pamplona, disponible en http://www.turismodepamplona.es/img/baluarte_1g.jpg

Figura 10

PALACIO DE CONGRESOS YAUDITORIO BALUARTE

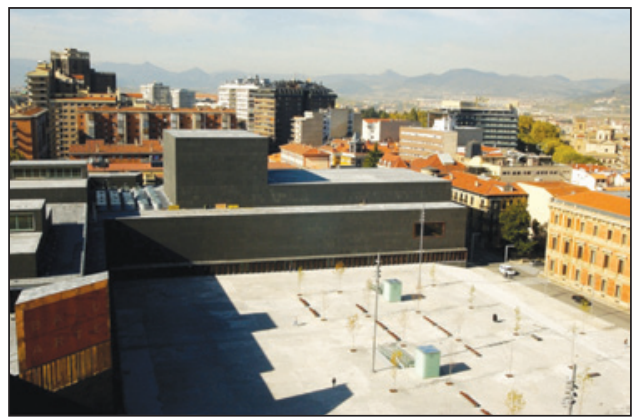

Fuente Ayuntamiento de Pamplona, disponible en http://www.turismodepamplona.es/img/baluarte_3g.jpg

No obstante, respecto a la revitalización del centro histórico, se han perdido importantes oportunidades. Una de ellas podría haber sido la ubicación en este espacio de la Universidad Pública de Navarra reutilizando antiguos contenedores infrautilizados, como se hizo en Toledo (Alli, 2001). Pero no ha sido la única oportunidad perdida. La falta de acuerdo entre el Gobierno de Navarra y el Ayuntamiento hizo que se descartaran varias ubicaciones, como el Palacio del Condestable, por ejemplo, para Biblioteca General, hoy situada en la plaza de San Francisco, en el antiguo Grand Hotel. Aunque se han realizado algunas intervenciones de cambio de uso en edificaciones infrautilizadas como el Departamento de Presidencia, Administraciones Públicas e Interior, el Departamento de Economía y Hacienda, el Departamento de Educación y el Departamento de Fomento y Vivienda, entre otros, no se han sabido aprovechar las potencialidades que el patrimonio monumental ofrecía para la instalación de nuevos usos relacionados con la administración, y la mayoría de las actuaciones realizadas no están ubicadas en el centro histórico sino en edificios del primer y segundo ensanche próximos al casco (figura 11). 


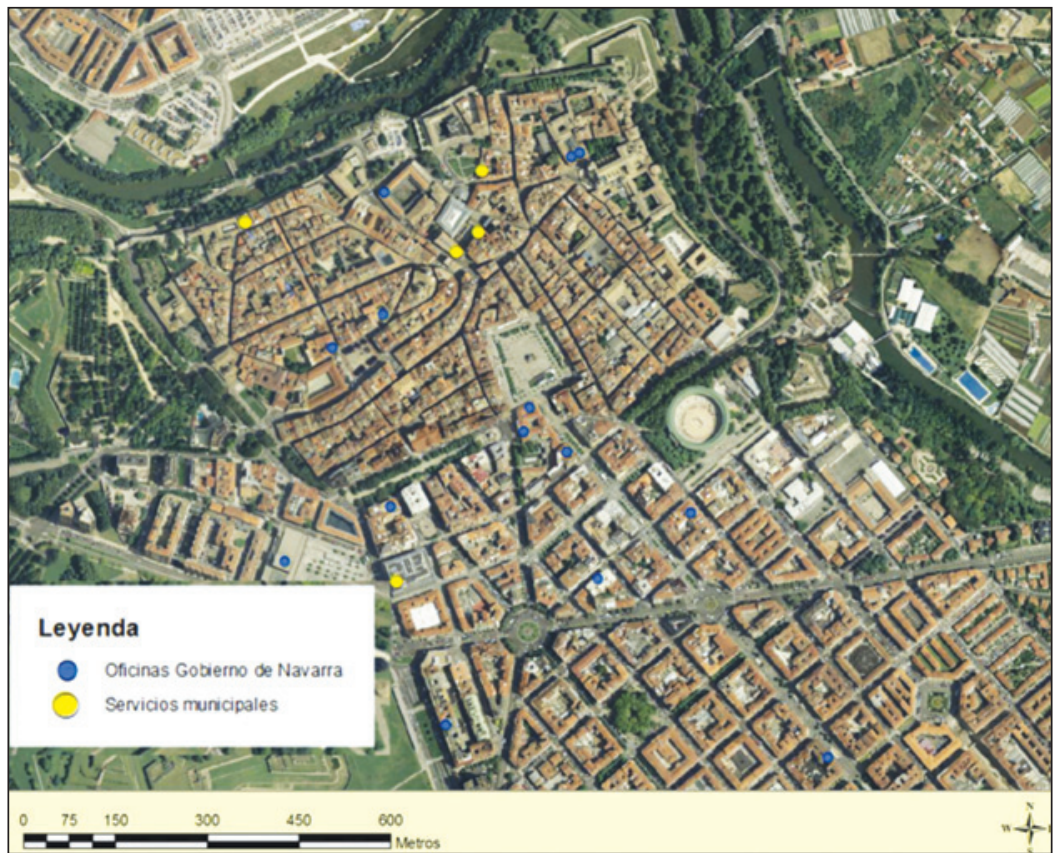

Fuente: elaboración propia.

Según Martínez Lorea (2010), la amenaza de una terciarización excesiva de su centro histórico también pende sobre Pamplona, no tanto por una alta concentración de locales pertenecientes a la Administración, sino por una alta concentración de entidades bancarias en el Paseo Sarasate, la localización de hoteles de cuatro estrellas en edificios históricos, tales como el Palacio de los Condes de Guenduláin o el antiguo Convento de Adoratrices, la instalación en el año 2003 de El Corte Inglés en el límite del barrio -que marca un desplazamiento de la referencia comercial clásica del centro histórico hacia el Ensanche donde la Avenida Carlos III se ha convertido en un auténtico paseo comercial-, y la apertura de nuevos locales hosteleros de tipo cafeterías y restaurantes como consecuencia de un cambio en la legislación, cambio que no se produce en relación con los bares nocturnos cuya apertura sigue estando prohibida. Se prevé asimismo la construcción y habilitación de un conjunto de espacios capaces de albergar instalaciones de carácter cultural y turístico destinadas a la proyección nacional e internacional de la ciudad de Pamplona, así como servicios vinculados a dichas instalaciones.

Pamplona busca combinar la imagen de ciudad histórica con la de ciudad asociada a la investigación. Estos planteamientos deben inscribirse en el interés actual de las ciudades por estar presentes en escenarios de repercusión internacional que les permitan transmitir su propia imagen. En el caso de Pamplona, el centro histórico cobrará así nueva importancia tanto por su recuperación y revalorización física y social cuanto por su conversión en referente y conden- 
sador de determinados valores. A lo largo de las últimas décadas hemos podido apreciar una apuesta de la administración local por rehabilitar el casco histórico, dando respuesta a los déficits que venía arrastrando desde hacía largo tiempo, pero también a través de la conformación del centro como un elemento vinculado a las estrategias de competitividad interurbana.

\section{IV.2. Toledo}

De forma paralela Toledo se convierte en capital de Castilla-La Mancha en 1982, actuando esta circunstancia como palanca de renovación, de recuperación urbana y de crecimiento (Campos, 2002), así como trampolín para un nuevo período de crecimiento y desarrollo para la ciudad (Ganau y Vilagrasa, 2003).

En lo relativo a las políticas urbanas puestas en marcha, la Junta de Comunidades de Castilla-La Mancha, y más en concreto la Consejería de Educación y Cultura, elaboró en 1989 el programa «Castilla-La Mancha a Plena Luz». A Plena Luz es un programa integral articulado en torno a cinco subprogramas: «Redacción de un Plan Especial», «Rehabilitación de Viviendas», «Mejora del Paisaje Urbano», «Potenciación del Equipamiento Sociocultural» y «Restauración Monumental» (Peris, 1993). Es el programa «Equipamiento Sociocultural» el que incluye las obras que presentan especial utilidad social, cultural, la recuperación de edificios para usos culturales y administrativos -públicos o privados-, y el equipamiento público en general. El programa «Restauración Monumental» está orientado a preservar los hitos de la imagen urbana y adecuar el patrimonio monumental a las necesidades actuales de la ciudad, introduciendo en algunos casos nuevos usos en los inmuebles rehabilitados.

Los redactores del Plan Especial del Casco Histórico de Toledo apuestan por la recuperación y puesta en valor de lo existente, tanto si se trata de monumentos importantes como de tejidos urbanos sin arquitectura de gran valor, pero que aportan ambientes y texturas interesantes. Esta cultura debe permitir una estrategia funcional basada en actividades compatibles con el casco histórico que admite el uso mixto de residencia y servicios y que asegure el mantenimiento de la singularidad monumental de Toledo con un rol activo de centro de ciudad de un hinterland territorial amplio (Peris, 2006). Siendo las dependencias administrativas estrictamente necesarias para las funciones de capitalidad, institucionales, representativas, culturales y educativas, y ante la necesidad de edificios para acoger los distintos organismos regionales se optó, en consonancia con el urbanismo de recuperación de la época, por abordar distintos procesos de rehabilitación en edificios histórico-artísticos del casco histórico que se encontraban en una lamentable situación, y asignarles un nuevo uso. A mediados de la década de los 80 empiezan a generalizarse procesos de recuperación de viejos contenedores infrautilizados cuyo objetivo es la ubicación en ellos de usos administrativos relacionados con la nueva situación de Toledo como capital de la región castellano-manchega. La reutilización de edificios singulares sin apenas uso para nuevas funciones permite la rehabilitación y conservación de estos edificios y la presencia de actividad en su entorno con todos los aspectos positivos que ello conlleva para la ciudad.

El objetivo fundamental de la rehabilitación es volver a reintegrar el edificio en la historia y hacer que vuelva a ser útil siendo el mismo edificio. En este sentido, Rafael Moneo (2012) considera que un edificio que deja de ser útil deja de ser arquitectura. Es evidente la capacidad de estos edificios para albergar usos distintos siempre que se respeten las carac- 
terísticas básicas de los mismos. El problema puede aparecer cuando se intenta aplicar la -en muchos casos excesiva- normativa actual a un edificio histórico con parámetros de otras épocas. La aplicación estricta de dicha normativa podría llevar, en algunas ocasiones, a la alteración de aspectos básicos de su formalización arquitectónica o incluso a la imposibilidad de su utilización para usos actuales ${ }^{5}$

En esta línea de actuación se han llevado a cabo por parte de la Junta de Comunidades importantes actuaciones de rehabilitación de edificios singulares, que ahora sirven como dependencias de sus propios servicios y como sede de instituciones culturales y educativas (Campos, 1991). Algunos ejemplos de intervenciones de rehabilitación para usos administrativos son, entre otros, el Palacio de Fuensalida para sede del gobierno regional, el Convento de San Gil para sede de las Cortes de Castilla-La Mancha, el Hospital del Nuncio para las Consejerías de Economía y Hacienda y Obras públicas y el edificio de Doncellas Nobles para Consejería de Educación y Cultura, si bien en la actualidad dicha Consejería se ubica en un edificio de nueva planta en el polígono de Santa María de Benquerencia.

De esta forma la capitalidad autonómica ha sido un elemento importante en la revitalización del conjunto histórico. Por otra parte, la presencia institucional incrementa la dinámica cultural y turística de la ciudad propiciando la recuperación de edificios monumentales para usos culturales. Entre otros, se rehabilita el Teatro de Rojas, la mezquita de Tornerías para albergar el Centro Regional de Artesanía, se llevan a cabo diversas obras de rehabilitación del Hospital de Tavera, siendo la más significativa la que plantea la ubicación en una de las plantas del Archivo de la Nobleza, y se rehabilita la planta superior del Alcázar para albergar la Biblioteca Pública de Toledo (Peris, 2006) (figura 12).

El Palacio de Fuensalida, edificado a finales de la primera mitad del siglo XV, es el mejor exponente palaciego del mudéjar toledano, donde se fusionan tres estilos: gótico, plateresco y mudéjar ${ }^{6}$. Declarado monumento histórico en 1962, fue adquirido en 1964 por la Dirección General del Patrimonio Artístico que encarga su restauración y lo abre al público en 1969, hasta 1983, momento en que se acondiciona para convertirse en la sede de la Presidencia de la Junta de Comunidades de Castilla-La Mancha (figuras 13 y 14). Dos décadas después se alertó del peligro de derrumbe tras la excesiva carga administrativa que sufría. Así, en agosto de 2007 comenzaron las obras de rehabilitación y, tras su inauguración en la primavera de 2010, se decidió que quedase abierto al público. El Palacio de Fuensalida se convierte así en un espacio de representación para la Comunidad Autónoma de Castilla-La Mancha y un espacio cultural para los castellano-manchegos. Además de ser los artífices de la última intervención en el Palacio de Fuensalida, Agustín Mateo y Mario Muelas son los autores de actuaciones tan emblemáticas para los castellano-manchegos como las reconversiones del convento de San Pedro Mártir y su ampliación en el convento de Madre de Dios, la rehabilitación del Palacio de Padilla para la instalación en él de la Facultad de Humanidades, o la actuación en la antigua Casa de la Moneda para convertirla en sede del Consejo Consultivo en Toledo.

5 Castilla-La Mancha. Revista de Información de la Junta de Comunidades de Castilla-La Mancha. Martes, 22-5-2012. Disponible en http://pagina.jccm.es/revista/sitio/index.php?option=com_content\&view=article \&id=269 $8 \&$ Itemid $=2473$

6 Palacio de Fuensalida: De la Aristocracia a la Democracia. Dossier de prensa.www.jccm.es/.../Dossier\%20 del\%20Palacio\%20de\%20Fuensalida.pdf 
El edificio de San Gil, originariamente llamado Convento de Franciscanos Descalzos de San José y conocido popularmente como Los Gilitos, sirve de sede a las Cortes de CastillaLa Mancha desde el 31 de mayo de 1986. Su construcción, emprendida por los hermanos Juan y Francisco de Herrera, data de 1614. Situado en un lugar privilegiado al sudoeste del casco histórico toledano, junto al río Tajo, disfruta de unas excelentes perspectivas sobre los cigarrales, el Puente de San Martín y la judería toledana (figuras 15 y 16). Las diversas utilizaciones del edificio a lo largo de sus 370 años de existencia (cárcel provincial, cuartel de la Guardia Civil y parque municipal de bomberos) produjeron un grave deterioro de su estructura. Su restauración y adaptación para sede parlamentaria se llevó a cabo por el arquitecto Fernando Chueca respetando las trazas originales del edifico y asumiendo su papel de símbolo autonómico, símbolo que constituye, junto con el escudo y la bandera de la Región, una de las señas de identidad de Castilla-La Mancha.

\section{Figura 12}

LOCALIZACIÓN DE LOS EDIFICIOS DEL GOBIERNO REGIONAL, DE LA UCLM Y LOS PRINCIPALES CONTENDEDORES CULTURALES EN EL CASCO HISTÓRICO DE TOLEDO

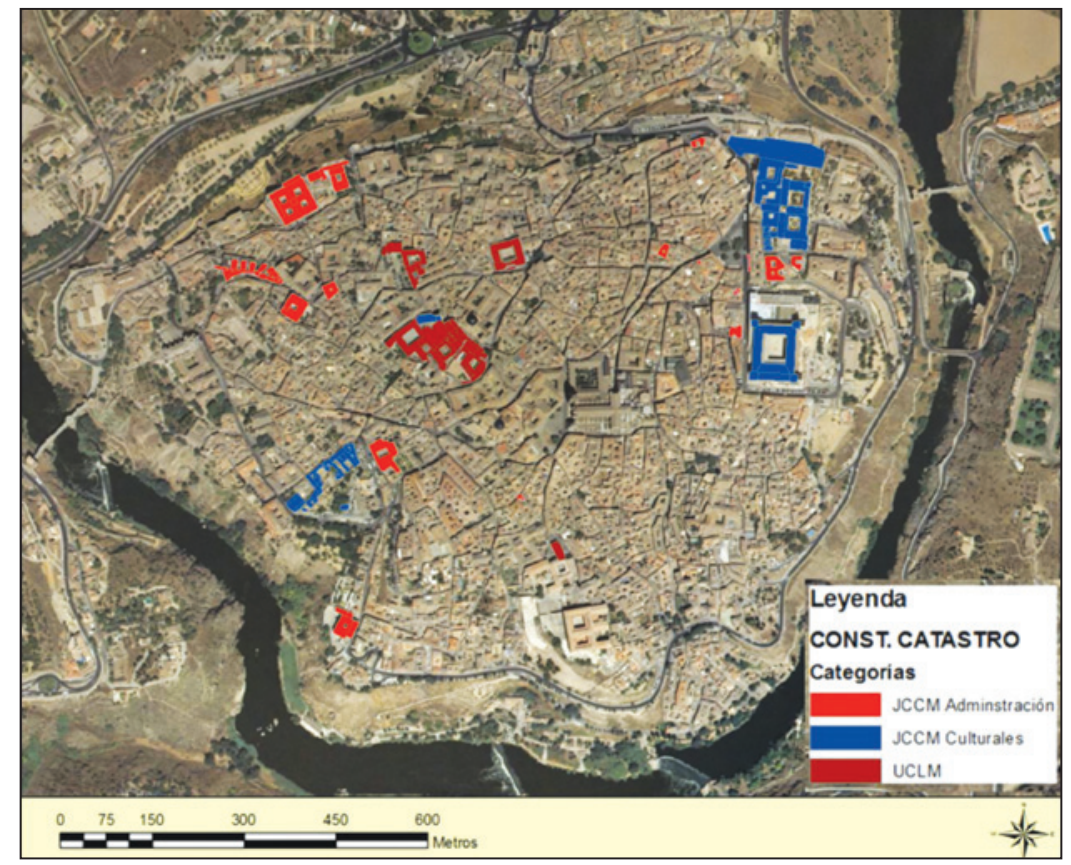

Fuente: elaboración propia.

En otro orden de cosas, la ciudad y la universidad obtienen beneficios mutuos. La universidad es muy importante en la recuperación de los centros históricos, como se demuestra en la experiencia europea (Peris, 2006); como afirma Lourdes Campos (2006), «hoy en día las políticas universitarias y urbanísticas compatibilizan la rehabilitación de los viejos edificios históricos con el fin de regenerar los cascos antiguos» (...); «tradicionalmente, la 
presencia de actividad universitaria ha aportado a los centros históricos mayor vitalidad que cualquier otro factor o equipamiento singular, al favorecer la recuperación del patrimonio, frenar la degradación, enriquecer la vida urbana con actividades de extensión universitaria y reactivar el mercado inmobiliario, entre otros aspectos». Ejemplos de ciudades donde se han puesto en marcha de forma paralela y complementaria ambas políticas son Oxford, Cambridge, Bolonia, Lovaina y Coimbra, o Salamanca, Alcalá de Henares y Santiago de Compostela en España.

Figura 13

PALACIO FUENSALIDAANTES DE LAREHABILITACIÓN

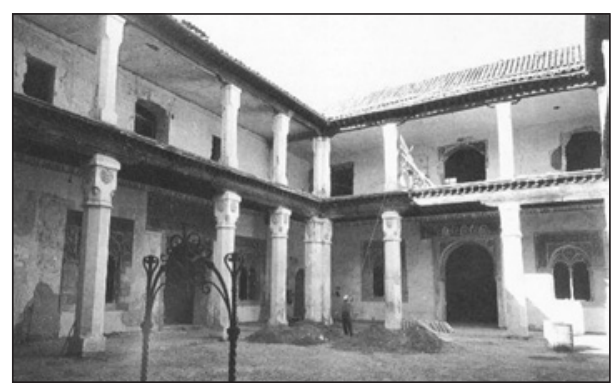

Fuente: www.castillalamancha.es

http://www.castillalamancha.es/sites/default/files/ Dossier\%20de1\%20Palacio\%20de\%20Fuensalida.pdf
Figura 14

PALACIO FUENSALIDA DESPUÉS DE LAREHABILITACIÓN

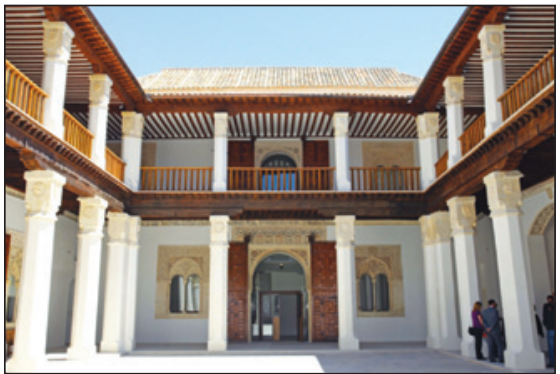

Fuente: www.castillalamancha.es

Origen: http://www.castillalamancha.es/sites/default/ files/documentos/fotografias/20130212/22100522 palacio_portada.jpg
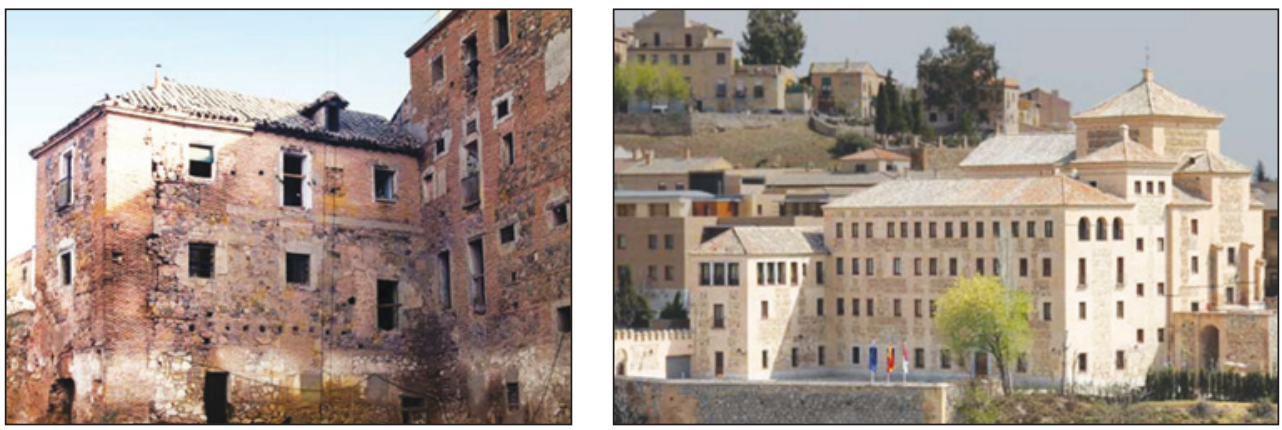

Fuente: CALVO, M. (coord..) (2008): Historia y Arte del Convento de San Gil. Cortes de Castilla-La Mancha.

Con el Estado de las Autonomías llega a Toledo una nueva situación para la institución universitaria. La Asociación de Amigos de la Universidad reclamaba un papel prioritario para el entonces Colegio Universitario. En junio de 1982 se publica la ley que reconoce el nacimiento de la Universidad de Castilla-La Mancha con el Rectorado ubicado en Ciudad 
Real y los vicerrectorados en Albacete, Cuenca y Toledo. La integración real del Colegio Universitario de Toledo en la Universidad de Castilla-La Mancha se producirá en 1990 aunque el Patronato del Colegio Universitario continúa su actividad hasta 1994. Con la cesión de San Pedro Mártir en 1993 se consolida un núcleo de edificios históricos que acogen las enseñanzas universitarias en Toledo (Peris, 2006).

Las Facultades y Escuelas Universitarias del Campus de Toledo se encuentran en emblemáticos edificios históricos, cosa que no ocurre en Pamplona donde la Universidad Pública de Navarra y la Universidad Privada se sitúan en la periferia de la ciudad. El campus de Toledo está dividido en dos zonas diferenciadas: el campus del casco histórico y la antigua Fábrica de Armas. En el casco histórico se imparten las titulaciones relacionadas con las Ciencias Sociales y las Humanidades. La Facultad de Ciencias Jurídicas y Sociales tiene su sede en el Antiguo Convento de San Pedro Mártir y en el Antiguo Convento de Madre de Dios. La Facultad de Humanidades tiene su sede en el Palacio de Padilla. El Palacio del Cardenal Lorenzana es la sede del Vicerrectorado del Campus y de Relaciones Institucionales y alberga el Centro de Documentación Europea y el Centro de Estudios de Consumo de la UCLM. Igualmente, la Universidad de Castilla-La Mancha posee un Centro de Investigación dirigido a las lenguas árabe y hebrea: la Escuela de Traductores, que toma su nombre de la célebre institución medieval homónima.

El Convento de San Pedro Mártir, construido en el siglo XIII, fue ensanchado y casi reedificado de nuevo bajo las trazas de Alonso de Covarrubias a mediados del siglo XVI. Tras la desamortización, fue utilizado como museo, panteón de toledanos ilustres y como centro de beneficencia. Desde 1969 viene funcionando como sede universitaria, primero como colegio universitario adscrito a la Universidad Complutense y, desde 1991, como la Facultad de Ciencias Jurídicas y Sociales de Toledo tras abordar diversas reformas entre los años 1992 y 1993, que integraron el edificio como parte de la Universidad de Castilla-La Mancha (figura 17). El Convento de la Madre de Dios, fundado en 1482 comienza su deterioro a partir de finales del siglo XVII, deterioro que continuará durante los siglos XVIII y XIX. Tras varias restauraciones parciales durante el siglo XX, la definitiva rehabilitación del convento se produce entre el año 2002 y el 2005 después de que la Universidad de Castilla-La Mancha adquiriera el inmueble para complemento del centro universitario de San Pedro Mártir y sus espacios administrativos (figura 18). El Palacio Padilla, casa nobiliaria gótica del siglo XV, cuyos elementos más destacados son su portada y el patio típico toledano, se remodela en 1985 para el Consejo Superior de Protección de Menores. Cedido el edificio a la Universidad de Castilla- La Mancha, en 1998 se rehabilitará según planos de los arquitectos Mario Muelas y Agustín Mateo (figura 19).

Ante la escasez de espacio, el palacio del Cardenal Lorenzana, realizado a finales del siglo XVIII, cuenta con una doble escalinata pegada a la fachada para hacer una entrada digna desde el primer piso, donde se encuentra la grandiosa portada de cinco vanos con monumentales columnas jónicas y decoración escultórica, que dan paso a un patio columnado. Actualmente es la sede del Vicerrectorado del Campus de Toledo y de Relaciones Institucionales de la Universidad de Castilla La Mancha (figura 20). El Palacio del Rey Don Pedro, edificio mudéjar del siglo XIV (figura 21), es en la actualidad sede de la Escuela de Traductores de Toledo, un centro de investigación dependiente de la Universidad de CastillaLa Mancha en el que se preparan traductores de árabe y hebreo, además de impartir otros cursos en estos idiomas. Todas estas instalaciones universitarias contribuyeron a la rehabi- 

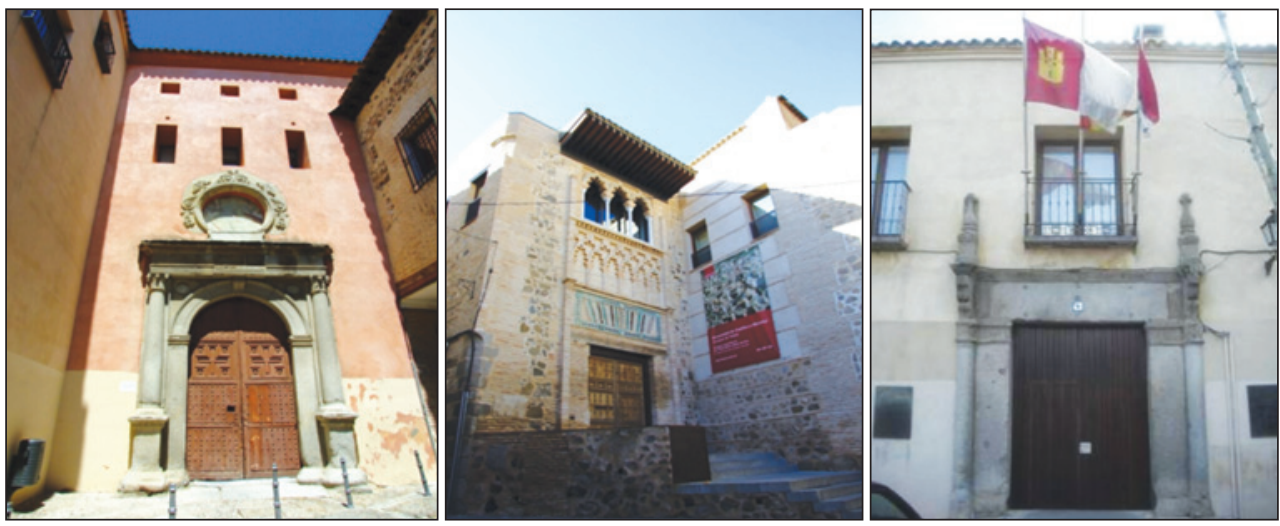

Fuente: ttp://www.monumentalnet.org/castilla_la_mancha/toledo/toledo/toledo/palacio_de_padilla.php?vis=2

litación de edificios monumentales antiguos y a la revitalización de la zona norte del centro histórico, zona conventual afectada de mayor despoblamiento y con contenedores de gran tamaño desocupados a la espera de rehabilitación y destino de un uso.

Otra parte del campus toledano se encuentra ubicado en la antigua Fábrica de Armas, la «otra ciudad histórica» que denomina Peris (2006), auténtica ciudad industrial de los siglos XIX y XX junto al río Tajo. Aquí se imparten las titulaciones relacionadas con las Ciencias Experimentales y de la Salud: Ciencias del Deporte, Ciencias del Medio Ambiente, Ingeniería Técnica Industrial, Enfermería, Fisioterapia y Magisterio. La recuperación del conjunto de la Fábrica de Armas para su ocupación por la Universidad de Castilla-La Mancha supuso un símbolo de progreso y desarrollo para la ciudad de Toledo (figura 22). Su origen se remonta a la renovación del país de la mano de Carlos III y al proyecto de Sabatini. En un principio era un único y gran edificio, ampliándose su número hasta los cincuenta, según las distintas épocas y necesidades industriales, dando como resultado lo que se denomina «Ciudad Industrial», de estilo neomudéjar, uno de los conjuntos mejor conservados y de mayor calidad de la arquitectura industrial realizada en España en los dos últimos siglos.

A partir de los años 60 la Fábrica de Armas empieza a quedar obsoleta y el número de trabajadores se va reduciendo durante las siguientes décadas, hasta su definitivo cierre en 1996. En 1998 el Ministerio de Defensa vende al ayuntamiento de Toledo los terrenos y edificios de la Fábrica de Armas y éste a su vez cede el uso a la Universidad de Castilla-La Mancha, la cual creó en esta zona de la ciudad el Campus Universitario Tecnológico de Toledo. Las actuales instalaciones universitarias ocupan más de una docena de los edificios restaurados y rehabilitados, resaltando entre todos ellos el Edificio Sabatini. Sus pabellones albergan laboratorios de docencia e investigación, aulas, seminarios, oficinas universitarias, bibliotecas y zonas deportivas para la actividad docente universitaria.

Pero la política de renovación y cambio de uso no solo se ha centrado en la potenciación de usos administrativos y universitarios en el casco, también hay que mencionar la poten- 


\section{Figuras 20, 21 y 22 \\ EDIFICIOS DE LA UNIVERSIDAD DE CASTILLA-LA MANCHA: \\ PALACIO DEL CARDENAL LORENZANA, PALACIO DE PADILLA Y FÁBRICA DEARMAS}
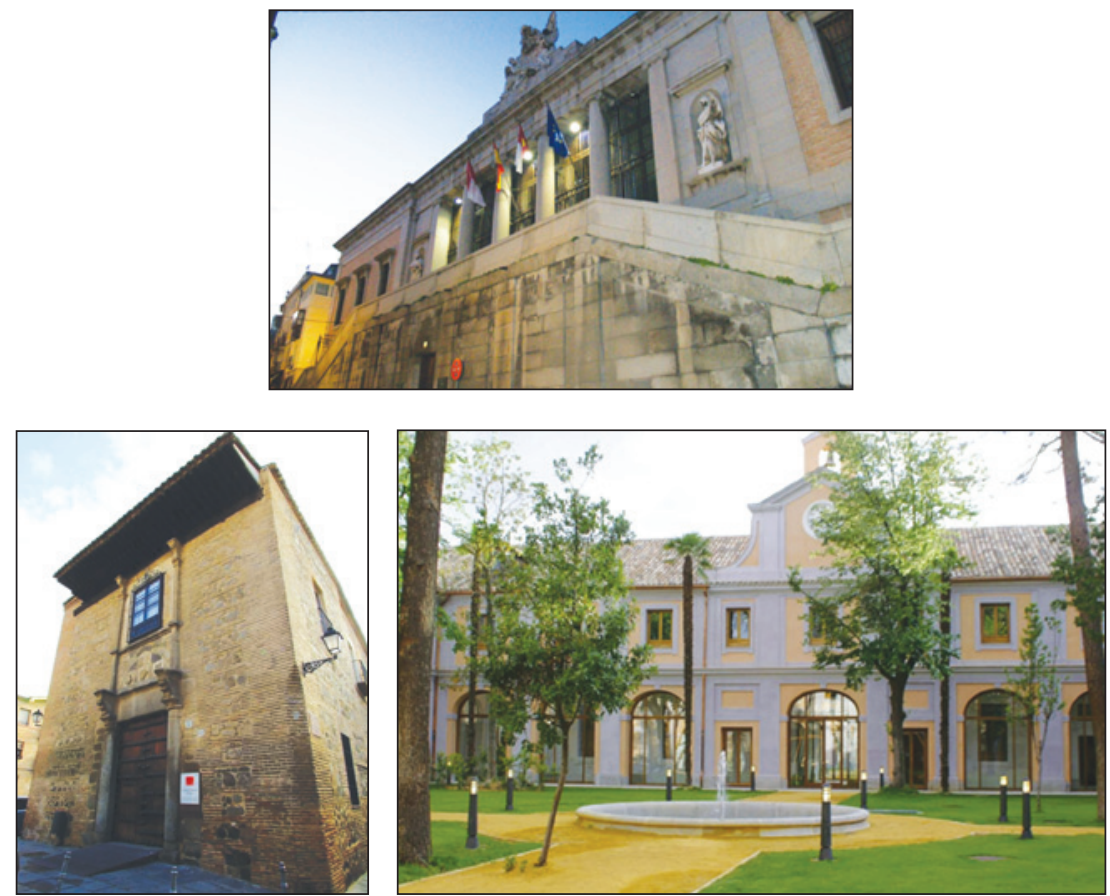

Fuente: http://www.toledo-turismo.com/turismo/contenido/mas-toledo/toledo-futura/ciudad-universitaria/palaciolorenzana.aspx; http://www.unaventanadesdemadrid.com/toledo-iv.html;

ciación de los usos culturales. Antiguas iglesias se han convertido en salas de conciertos, un cementerio de monjas o un antiguo convento en centro cultural... Este es el caso del centro cultural San Marcos. En los años 90 se acomete por parte del Ayuntamiento uno de los más emblemáticos proyectos del Programa de Renovación Urbana del Centro Histórico de Toledo: la rehabilitación y recuperación del convento de San Marcos para albergar en sus dependencias el Archivo Municipal de nueva planta y el Centro Cultural (figura 23). El proyecto surgió con el objetivo de recuperar para la vida ciudadana uno de los mayores edificios de la ciudad, preparándolo y adaptándolo para un uso de nueva creación que permitiese centralizar la realización de actividades culturales: archivo, biblioteca, sala de exposiciones, auditorio, etc. Fundado inicialmente por los trinitarios en el siglo XIII, fue desalojado por las sucesivas desamortizaciones estatales hasta convertirlo en cuartel de Infantería y, después, en dependencias municipales secundarias. Tras los destrozos que dejó en su estructura la guerra civil, el deterioro y el abandono se extendieron a un edificio imponente desacralizado y vaciado de cualquier contenido. Últimamente ha sido taller municipal de carpintería y almacén de enseres y carrozas. El Plan Especial del Casco Histórico proponía la recuperación y rehabilitación de San Marcos para dotarle de un uso cultural y de ocio 
de gran valor, tanto para los residentes en la zona monumental como para todos los toledanos y visitantes, actuación que, como ya se ha comentado, se llevó a cabo en los años 90.

También relacionada con la función cultural, debemos señalar la actuación de la Biblioteca Regional en la segunda planta del Alcázar toledano, reconvirtiendo el tradicional uso militar en cultural, aún a pesar de compartir la Biblioteca el espacio con el antiguo Museo del Asedio en la primera planta del Alcázar, y con el Museo del Ejército (Campos Romero, 1998), aunque tras la aprobación de la Ley de Memoria Histórica y la inauguración del nuevo Museo del Ejército de Tierra, tal instalación ha desaparecido.
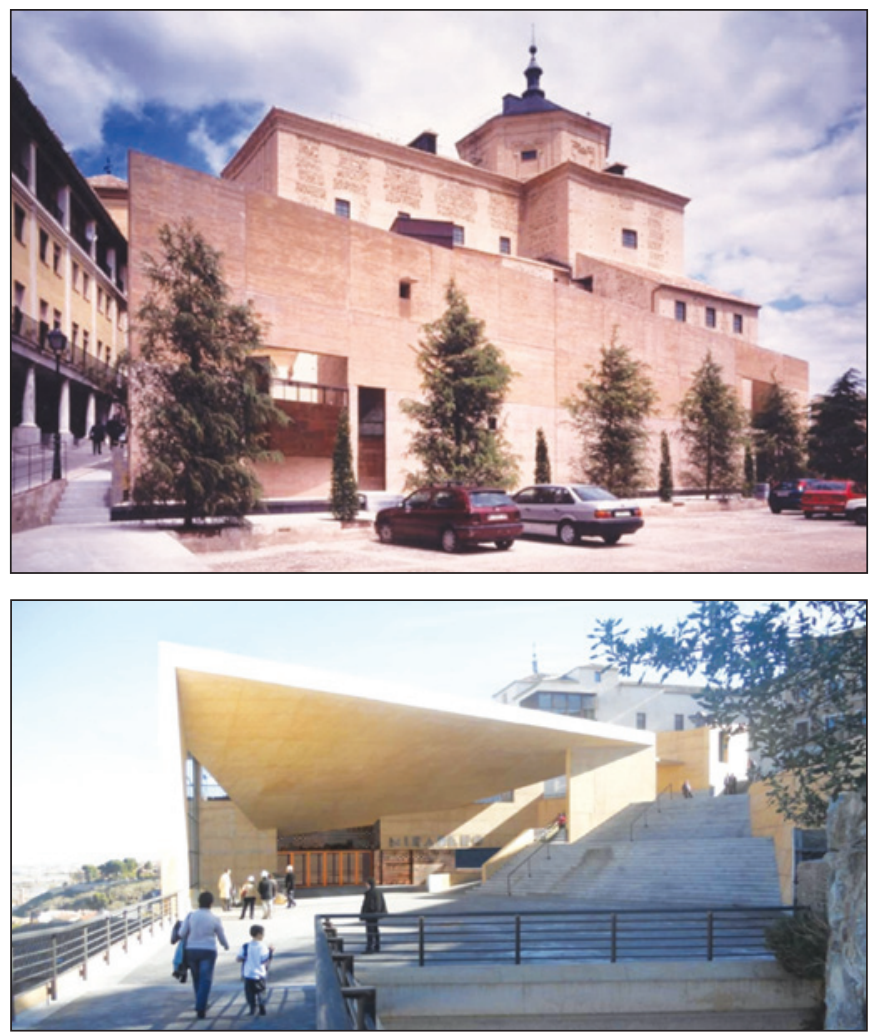

Fuente: Urbanscraper Arquitectura + Urbanismo + Ingeniería. Disponible en http://www.urbanscraper.com/2007/04/ futuro-palacio-de-congresos-de-toledo.html; http://www.turismocastillalamancha.com/que-hacer/reuniones-incentivos/sedes-eventos/toledo/toledo/palacio-de-congresos-de-toledo/\#a

El proceso de creación de la Biblioteca de Castilla-La Mancha en el Alcázar se inició en septiembre de 1986, fecha en la que el Ministerio de Defensa transfirió a la Junta de Comunidades una parte del edificio para usos culturales. En 1989, las Cortes regionales aprobaron la Ley de Bibliotecas de Castilla-La Mancha que incluía en dos de sus artículos las funciones 
de la futura Biblioteca regional. Otros dos acuerdos firmados por el presidente de la región y los ministros de Cultura (1990) y Defensa (1991) significaron el paso definitivo para la instalación de los fondos de la Biblioteca Pública de Toledo en el Alcázar, cuyas obras de acondicionamiento, dirigidas por José María Pérez -Peridis-, fueron adjudicadas en 1994 y concluidas cuatro años después, en 1998.

Para terminar con los ejemplos de intervenciones culturales en el centro histórico de Toledo, mencionar una obra nueva: el Palacio de Congresos El Miradero, ubicado sobre un escarpe rocoso dominando el río Tajo y su vega. Obra vanguardista de Rafael Moneo, se integra en la ciudad histórica con el más absoluto respeto hacia el paisaje y el entorno monumental en el que se ubica (figura 24). Se configura como una auténtica extensión del corazón del Casco Histórico, de la Plaza de Zocodover y como nuevo pulmón para la zona histórica pues sobre la cubierta general del edificio, se soportan los Jardines del Miradero ${ }^{7}$.

En definitiva, la decisión de instalar la capital de la región en Toledo ha actuado como dinamizador y refuerzo de sus funciones centrales en materia administrativa, que se traduce en la localización generalizada de consejerías en grandes edificios recuperados y de la misma sede de la Presidencia Regional en el Palacio de Fuensalida, así como en materia docente y cultural.

\section{CONCLUSIONES}

Durante estos años ha tenido lugar un interesante proceso de formulación e instrumentalización de políticas urbanas o, al menos de intervenciones, orientadas, en mayor o menor medida, a proteger, renovar, recuperar o revitalizar los centros históricos de nuestras ciudades. Como consecuencia de la aplicación de estas políticas se ha conseguido una importante recuperación física del patrimonio monumental y cambios funcionales relacionados con la utilización de ese patrimonio arquitectónico como dependencias de la nueva administración regional.

Los últimos años del siglo XX, con la ubicación de la capitalidad regional en Toledo, han supuesto un nuevo impulso revitalizador para sus edificios monumentales y un inicio de la recuperación de los espacios urbanos y edificios residenciales. A pesar de que en algunas ocasiones se ha calificado al programa A Plena Luz como de fachadista, hay que admitir que ha facilitado el cambio de uso en algunos inmuebles y ha producido una incipiente revitalización. Con las actuaciones del mencionado programa regional se está potenciando el centro histórico de Toledo como un centro fundamentalmente terciario, en relación con la instalación de nuevos usos relacionados con la nueva capitalidad. En definitiva, el centro histórico de Toledo continúa siendo el centro de actividad de la ciudad por su especialización en funciones administrativas, docentes, culturales y económicas de carácter terciario. Además, la función universitaria permite aprovechar el potencial del patrimonio monumental y edificado, incentivar la recuperación de viviendas para profesores y estudiantes, y generar el establecimiento de comercios de productos de primera necesidad y comercios especializados en la cultura como librerías y papelerías.

Por su parte, en Pamplona, las operaciones de revitalización del centro histórico no han estado asociadas a las funciones de capitalidad autonómica. Se han puesto en marcha

7 http://www.toledo-turismo.com/turismo/contenido/mas-toledo/ciudad-congresos/palacio-congresos.aspx 
algunas iniciativas como la Oficina de Rehabilitación y la aplicación de la Ordenanza Municipal de ayudas, iniciativas que se centran no tanto en el centro histórico sino fundamentalmente en el primer y segundo ensanche en las zonas más próximas al primero, y esto en relación con la estigmatización de que, según los redactores del Programa URBAN, es objeto el casco histórico por parte de la sociedad de Pamplona. De hecho, la mayor parte de los edificios administrativos están localizados en edificios históricos de los ensanches, aunque próximos al casco. En cuanto a la función universitaria, la creación de la Universidad Pública de Navarra en el año 1987 no provocó una potenciación de las operaciones de rehabilitación y cambio de uso en estos espacios urbanos, abordándose la construcción de nueva planta de toda la infraestructura inmobiliaria que habría de acoger estos usos en la periferia de la ciudad.

Para terminar, las ciudades históricas, con algunas significativas excepciones, son reacias a innovar en el terreno de la gestión. No obstante, la recuperación urbana de Toledo se ha consolidado gracias a la existencia de un foro de coordinación administrativa y cooperación institucional, la existencia de adecuados instrumentos urbanísticos y la progresiva implicación de los ciudadanos (Troitiño, 2003). El consorcio puede ser un instrumento adecuado para diseñar estrategias de futuro y consolidar alianzas que permitan la utilización del patrimonio cultural urbano como motor de desarrollo en el marco de estrategias de recuperación urbana integradas y de recuperación del patrimonio y cambio de uso del mismo.

\section{REFERENCIAS}

ALLI TURRILLAS, J.C. (2001): «El dilema de Pamplona: reurbanizar su casco urbano o urbanizar su cuenca». BIBLID, Azkoaga. n 8, 49-78.

ALMARCHA NÚÑEZ-HERRADOR, E.; DÍEZ DE BALDEÓN GARCÍA, P.; PERIS SÁNCHEZ, D.; SÁNCHEZ SÁNCHEZ, I. (2001): Paisajes de los Conjuntos históricos. Castilla-La Mancha. Ciudad Real. Centro de Estudios de Castilla-La Mancha. Junta de Comunidades de Castilla-La Mancha. 359 p.

ALTHUSSER, L. (1976): «Idéologie et appareils idéologiques d'Etat (Notes pour une recherché)», en Positions, Paris, Editions sociales, 1976, p. 67-125.

ANDERSON, B. (1996): L'imaginaire national: réflexions sur l'origine et l'essor du nationalisme, Paris, Éditions La Découverte, 212 p.

BOBBIO, L. (1992): Le politiche dei beni culturali in Europa, Bologne, Il Mulino, 261 p.

BRENNER, N. (2004): New state spaces: urban governance and the rescaling of statehood, Oxford, Oxford University Press, $351 \mathrm{p}$.

BUSQUETS, J. (2000): Toledo y su futuro. El Plan Especial del Casco Histórico de Toledo. Madrid. Ayuntamiento de Toledo, Caja de Castilla-La Mancha, Empresa Municipal de la Vivienda de Toledo, S.A.

CALVO, M. [coord.] (2008): Historia y Arte del Convento de San Gil. Toledo. Cortes de Castilla-La Mancha. 191p.

CAMPOS ROMERO, M.L. ( 2002): «Análisis del turismo en Castilla-La Mancha en el umbral del nuevo milenio» en CAMPOS ROMERO, M.L. (coord.), El turismo en Castilla-La Mancha : análisis y prospectiva. Cuenca. Ediciones de la Universidad de CastillaLa Mancha. Estudios n ${ }^{\circ} 83.69-102$. 
CAMPOS ROMERO, M.L. (1991): «Toledo A Plena Luz. Un programa de rehabilitación». Anales de Geografía de la Universidad Complutense, Vol. 11, 217-223.

CAMPOS ROMERO, M.L. (1998): Transformaciones del comercio minorista del centro histórico de Toledo, inducidas por los actuales cambios funcionales, en CAMPESINO FERNÁNDEZ, A. J. (dir.): Comercio, turismo y cambios funcionales en las ciudades españolas Patrimonio de la Humanidad. Cáceres. Cámara de Comercio e Industria. Disponible en http://www.camaracaceres.es/actividades/publicaciones/libros/completos/67/ contenidos/toledo.htm

CAMPOS ROMERO, Mª L Y OTROS (1988): «Toledo: Estudio geográfico-urbanístico del Casco Histórico» en Simposio Toledo ¿Ciudad Viva? Toledo ¿Ciudad Muerta?, Toledo, Colegio Universitario de Toledo, 3-74.

CORREDOR SIERRA, B. (2009): «Séptimo Catálogo Español de Buenas Prácticas». Boletín $C F+S 41$. Madrid. Instituto Juan de Herrera. Disponible en http://habitat.aq.upm.es/ boletin/n41/npres.html

DJAMENT-TRAN, G. (2013): «Patrimoine urbain, lieux de mémoire et fonctions de capitale à Berlin, de l'Unité allemande à la réunification», Revue Géographique de l'Est. [En ligne], vol. 53 / 3-4 I 2013, mis en ligne le 02 juillet 2014, consulté le 17 septembre 2014. URL: http://rge.revues.org/5034

GANAU CASAS, J. y VILAGRASA IBARZ, J. (2003): «Ciudades medias en España: posición en la red urbana y procesos urbanos recientes» en CAPEL, H. (coord.): Ciudades, arquitectura y espacio urbano. Alicante, Colección Mediterráneo Económico, Caja Rural Intermediterránea Sociedad Cooperaativa Cdto, Instituto de Estudios Socioeconómicos de Cajamar.

MARTÍNEZ LOREA, I. (2010): «El papel del civismo en la recuperación de los centros históricos». Congreso de Sociología «Treinta años de sociedad, treinta años de sociología». Universidad Pública de Navarra, Pamplona, 1, 2 y 3 de julio. Disponible en http://www. fes-web.org/congresos/X/grupos-trabajo/grupo.php?numero=5

MICOUD, A. (2005): «Patrimonialisation: redire ce qui nous relie? Un point de vue sociologique»; en Barrère C. et alii, Réinventer le patrimoine. De la culture à l'économie, Paris, L'Harmattan, p. 81-96.

ORDEIG-CORSINI, J.M., RIVES-NAVARRO, L., ZAMARBIDE-URDÁNIZ, A. y GORTARI-CHAMORRO, C. (2011): «Pamplona. Desarrollos urbanos 1950/2000. Catálogo» en I Jornadas sobre urbanismo en el norte de España. Disponible en http://dspace.unav. es/dspace/handle/10171/18642

PERIS SÁNCHEZ, D. (1993): «La modificación de la ciudad «Castilla-La Mancha a Plena Luz» en La recuperación del Patrimonio Histórico de los Centros Rurales. Sigüenza. Universidad de Alcalá Vicerrectorado de Extensión Universitaria. 26 p.

PERIS SÁNCHEZ, D. (2006): La modificación de la ciudad: restauración monumental en Toledo s. XIX y XX. Tesis Doctoral Departamento Construcción y Tecnología Arquitectónica. E.T.S. Arquitectura (UPM)

SPATE, O. H. K. (1942): «Factors in the development of capital cities», The Geographical Review, $\mathrm{n}^{\circ} 3$, julio 1942, p. 622-31.

TROITIÑO VINUESA, M.A. (2003): «La protección, recuperación y revitalización funcional de los centros históricos» en CAPEL, H. (coord..) Ciudades, arquitectura y espacio 
urbano. Colección Mediterráneo Económico. Caja Rural Intermediterránea. Sdad. Coop. Cdto. Instituto de Estudios Socioeconómicos de Cajamar $\mathrm{n}^{\circ} 3$. Disponible en $w w w$ fundacioncajamar.es/mediterraneo/revista/me0308.pdf

TROITIÑO VINUESA, M. A. (2000): «Ciudades históricas y turismo: los desafíos de la sostenibilidad». Ciudad y Patrimonio. Revista de Arqueología, Arte y Urbanismo, $\mathrm{n}^{\circ} 4$, 93-108.

VOISIN, C. (2007): «Le centre, la mémoire, l’identité. Des usages de l'histoire dans la (re)-construction du Nouveau marché de Dresde», Espaces et sociétés, 3/2007, n 130, p. 87-101.

ZÁRATE MARTÍN, M.A. (2007): «Toledo. Planeamiento y especulación en ciudades históricas». Anales de Geografía, vol. 27, nº 2, 151-175.

\section{RECURSOS WEB}

- BARROS, P. Biografía de Carlos V. http://www.librosmaravillosos. com/biografia\% 20Carlos \%20V/capitulo11.html

- Castilla-La Mancha. Revista de Información de la Junta de Comunidades de CastillaLa Mancha. Martes, 22-5-2012.Disponible en http://pagina.jccm.es/revista/sitio/index. php?option=com_content $\&$ view=article $\&$ id=2698\&Itemid=2473

- Diariodenavarra. es. Especiales. Arquitectura en Pamplona. Disponible en http://www. diariodenavarra.es/especiales/arquitectura/index .asp?sec=anos 90

- Dirección General de Cultura. Departamento de Cultura, Turismo y Relaciones Institucionales. Navarra.es. Baluarte, palacio de Congresos y Auditorio de Navarra. http://www. turismo.navarra.es/esp/propuestas/congresos/desarrollo/baluarte.htm

- El Gobierno de Navarra edita el libro «El Palacio Real de Pamplona» sobre la historia de la sede del Archivo General. Martes, 14 de diciembre de 2004. Disponible en http://www. navarra.es/home_es/Actualidad/Sala+de+prensa/Noticias/2004/12/14/1412cu60.htm.

- España Vista Panorámica, Toledo. by Talavan panoramio.com. Disponible en https:// www.google.es/search? $q=$ Espa\%C3\%Bla + Vista + Panor\%C3\%Almica,+ Toledo.+ by $+T$ alavan + panoramio.com $\&$ biw $=1920 \& b i h=920 \&$ tbm $=i s c h \&$ tbo $=u \&$ source $=$ univ \& $s a=X$ \&ei=7T8EVcP_KY71aq-LgpgP\&ved=0CCAQsAQ

- Geoperspectivas. Marzo 2011. Disponible en ttp://geoperspectivas2bachiller.blogspot. com.es/2011/03/toledo-como-aquellas-ciudades-con-una.html

- http://parlamento.eleaweb.com/index.php?idi=cas\&sec=5\&sub=16

- http://parlamento.eleaweb.com/index.php?idi=cas\&sec=5\&sub=16

- http://www.iesnavarrovilloslada.com/public/proyectos/comenius/pamplona.index_Archivos/urbanismo.htm

- http://www.navarra.es/home_es/Actualidad/Sala+de+prensa/Noticias/2004/12/14/1412cu60. htm

- http://www.navarra.es/home_es/Actualidad/Sala+de+prensa/Noticias/2004/12/14/1412cu60. htm

- http://www.navarratour.com/?page_id=681

- http://www.toledo-turismo.com/turismo/contenido/mas-toledo/ciudad-congresos/palaciocongresos.aspx 
- http://www.toledo-turismo.com/turismo/contenido/mas-toledo/toledo-futura/ciudad-universitaria/palacio-lorenzana.aspx;

- http://www.unaventanadesdemadrid.com/toledo-iv.html;

- Navarraconfidencial.com 27 de abril de 2012. Disponible en http://www.navarraconfidencial.com/2012/04/27/el-parlamento-de-navarra-aprueba-la-dacion-en-pago/

- Palacio de Fuensalida, en Toledo, sede del Gobierno de Castilla-La Mancha. lacerca.com. Disponible en http://www.monumentalnet.org/castilla_la_mancha/toledo/toledo/toledo/ palacio_de_padilla.php?vis=2

- Palacio de Fuensalida: De la Aristocracia a la Democracia. Dossier de prensa. $w w w . j c c m$. es/.../Dossier\%20del\%20Palacio\%20de\%20Fuensalida.pdf

- Plan Municipal de Pamplona. TEXTO REFUNDIDO. Aprobación Definitiva: Acuerdo C.O.T. 18/12/02 Memoria I - Análisis.

- turismocastillalamancha.com/que-hacer/reuniones-incentivos/sedes-eventos/ toledo/toledo/palacio-de-congresos-de-toledo/\#a http://www.flickr.com/photos/rlasaosa/6658992751/

- Urbanscraper Arquitectura + Urbanismo + Ingeniería. Disponible en http://www.urbanscraper.com/2007/04/futuro-palacio-de-congresos-de-toledo.html; http://www. 